\title{
The Role of Laparoscopy and Laparoscopic Ultrasound in Staging and in Palliation of Upper Gastro-Intestinal Malignancies: The Egyptian National Cancer Institute Experience
}

\author{
Zeiad S. Gad, Waheed Y. Gareer, Osama A. El-Malt, Hussein O. Soliman*, \\ Mohamed G. Abdel Menem, Maher H. Ibraheem \\ Surgical Oncology Department, National Cancer Institute, Cairo University, Cairo, Egypt \\ Email: *Soliman@daad-alumni.de
}

How to cite this paper: Gad, Z.S., Gareer, W.Y., El-Malt, O.A., Soliman, H.O., Menem, M.G.A. and Ibraheem, M.H. (2017) The Role of Laparoscopy and Laparoscopic Ultrasound in Staging and in Palliation of Upper Gastro-Intestinal Malignancies: The Egyptian National Cancer Institute Experience. Journal of Cancer Therapy, 8, 464471.

https://doi.org/10.4236/jct.2017.85040

Received: March 4, 2017

Accepted: May 23, 2017

Published: May 26, 2017

Copyright $\odot 2017$ by authors and Scientific Research Publishing Inc. This work is licensed under the Creative Commons Attribution International License (CC BY 4.0).

http://creativecommons.org/licenses/by/4.0/

\begin{abstract}
Background: Surgical resection of upper gastrointestinal malignancies occasionally carries substantial morbidity due to inaccurate pre-operative staging. The potential to prevent needless laparotomy by means of precise staging is the pouring force behind the use of diagnostic laparoscopy (DL). Objective: To assess the role of laparoscopy and laparoscopic ultrasound (LUS) in proper staging of upper gastro intestinal malignancies, and in potential palliation in advanced cases for pain (by neurolytic celiac plexus block) or gastric outlet obstruction (by laparoscopic bypass surgery). Study design: In this prospective study, 62 patients with lower esophageal, gastric and peri-pancreatic carcinomas were joined after written informed consent. All patients were examined with laparoscopy and LUS with the help of frozen section analysis to any doubtful metastatic site, peritoneal fluid and ascitic fluid analysis. Results: DL helped us to avoid needless laparotomy in $22.5 \%$ of patients, reducing its post-operative complications, hospital stay and cost. DL also helped us to do palliative management either in the form of gastric bypass or laparoscopic celiac plexus block. Conclusion: we praise the use of DL as a safe, effective and complimentary method to the other routine imaging modalities, in proper staging and palliation for upper gastrointestinal malignancies.
\end{abstract}

\section{Keywords}

Laparoscopic Palliation, Laparoscopic Ultrasound (LUS), Laparotomy, Staging Laparoscopy (SL), Upper Gastrointestinal Malignancies 


\section{Introduction}

Surgical resection of upper gastro-intestinal malignancies carries substantial morbidity and mortality. In many cases, these cancers are thought to be resectable until the phase of laparotomy, at which time widespread or metastatic disease is revealed, preventing resection [1].

The potential to avert a non-therapeutic laparotomy by means of precise and less aggressive staging is the pouring force behind laparoscopic staging of upper gastrointestinal malignancies. [2] Laparoscopy can play a complementary role in the pre-operative radiological examination in the precise staging of abdominal malignancies [3].

Precise staging supports in the suitable management choice for cure or palliation. Furthermore, research concerning neo-adjuvant protocols for locally advanced cancers makes accurate staging bossy. [4] Laparoscopic analysis can envisage the primary tumor, recognize hepatic metastases, detect regional nodal metastases and perceive small-volume peritoneal disease unnoticed by other non-invasive staging modalities, such as computerized tomography (CT), magnetic resonance imaging (MRI), or ultrasonography (US). The technique also eases obtaining biopsy specimens and aspiration cytology. [4] Laparoscopic ultrasound (LUS) can be used to assess deep organ parts that are not open to inspection, and can perceive hepatic metastases not noticed by the conventional pre-operative imaging such as US and CT [4] [5].

Sometimes, palliative measures such as laparoscopic gastric bypass for gastric outlet obstruction and laparoscopic celiac plexus block to treat stubborn pain from upper abdominal malignancies can be achieved [5] [6].

\section{Aim of the Work}

To assess the starring role of laparoscopy and LUS in:

1) Correct staging of upper gastro-intestinal malignant tumors [lower esophageal, gastric and peri-pancreatic (pancreatic head, peri-ampulary or distal common bile duct) carcinomas].

2) Potential palliation in advanced patients for pain (by neurolytic celiac plexus block) or gastric outlet obstruction (by laparoscopic bypass surgery).

\section{Patients and Methods}

This study was completed in the National Cancer Institute, Cairo University. 62 patients with pathologically confirmed either lower esophageal, gastric or peripancreatic malignancies were joined in the study.

All patients were candidates for proper staging using laparoscopy and LUS. Laparoscopic assisted biopsies were taken from any doubtful hepatic deposits, nodal metastasis or peritoneal deposits. Peritoneal fluid cytology or peritoneal wash were done in every case.

Data collected during laparoscopic staging were reviewed during open surgery in operable cases. Advanced and metastatic cases were candidates for laparos- 
copic palliation in the form gastric bypass in gastric outlet obstruction or celiac plexus block (CPB) in subjects with stubborn abdominal pain, particularly cases with advanced pancreatic carcinomas.

\subsection{Inclusion Criteria}

Patients with no contraindications for laparoscopy, no sepsis or active general infection, stage I, II, and III lower esophageal, gastric and peri-pancreatic carcinomas (for correct staging), cases with stage IV (for appropriate staging and probable palliation), and cases received neo-adjuvant chemotherapy or radiotherapy (to estimate the outcome of the management).

\subsection{Exclusion Criteria}

Patients who were unfit for surgery, or patients rejecting diagnostic laparoscopy, were excluded from the study. Patients with stage IV disease not pliable for palliation (not complaining of pain or appearance of gastric outlet obstruction) were also excluded, since there would be no symptoms we would be able to palliate.

All patients were subjected to history taking, physical examination, serum laboratory tests included; CBC, coagulation profile, full liver profile, renal profile and CEA, CA.19.9 as tumor markers), radiological investigations included; pelvi-abdominal CT and chest CT scan for exploration of the primary tumor and as metastatic work up, upper GI endoscopy and biopsy in lower esophageal and gastric carcinoma, ERCP and possible biliary stenting in peri-pancreatic carcinoma with malignant obstructive jaundice.

\section{Results}

This study comprised 62 patients with upper gastrointestinal malignancies, 41 (66\%) males and 21 (34\%) females with mean age $56.1 \pm 4.1$ years. Patients were further divided into 5 subgroups; 21 cases (33.9\%) with gastric carcinoma, 12 cases (19.4\%) with lower esophageal carcinoma, 12 cases (19.4\%) with pancreatic head carcinoma, 10 cases (16.3\%) with peri-ampullary carcinoma and 7 cases (11.3\%) with common bile duct (CBD) carcinoma.

Pre-operative CT scan (Figure 1) chest was completed in every subject and was free of metastatic deposits; tumor markers (CEA, CA19.9) were prepared in selected cases; and routine pre-operative examinations were normal in all cases. All data collected during staging laparoscopy (SL) were established after exploration of operable cases and were correct except in only 4 cases giving SL and LUS procedure specificity (77.8\%), positive predictive value $(91.8 \%)$, sensitivity $(100 \%)$ and $(100 \%)$ negative predictive value.

In these four cases the SMV was encased and invaded in one case of pancreatic carcinoma and one case of distal CBD carcinoma. The other two cases of gastric carcinomas showed fixed celiac and porta-hepatis nodes and one of them showed also para-aortic nodes, and a palliative gastrectomy was performed.

Peritoneal wash and cytology was completed in all cases without ascitis (53 
cases, 85.5\%) and all of them were negative for malignant cells, ascitic fluid cytology was taken in 9 cases and was positive for malignancy, nodal biopsy was taken in 8 cases and was positive, peritoneal biopsy in one case and was positive, omental biopsy in one case and was positive and liver biopsy from one case and was negative.

After diagnostic laparoscopy (DL), 14 (22.5\%) cases were inoperable and protected from unnecessary laparotomies and 48 (77.5\%) were operable. The established criteria of inoperability were; ascitis in 9 cases (14.5\%), nodal deposits in 8 cases $(12.9 \%)$, liver deposits in 5 cases $(8.1 \%)$, peritoneal deposits in one case (1.6\%) (Figure 2) and omental nodule in one case (1.6\%). Collective criteria were found in numerous of these inoperable cases.

Palliative management was done in only four cases (6.5\%); laparoscopic CPB in two cases, laparoscopic gastro-jujenstomy in one case and palliative laparoscopic gastrectomy and gastro-jujenstomy in one case.

The average hospital stay was two days for all subjects of DL only with $45 \mathrm{mi}-$ nutes (+/ -10 minutes) operational time. Minor complications were met in only 3 cases $(4.8 \%)$. One case with laparoscopic CPB complicated with transient postoperative hypotension, which was adjusted with intra-venous fluids, the second case with laparoscopic $\mathrm{CPB}$ complicated with self-limiting diarrhea for one day with transient hypotension, and the third case with laparoscopic gastro-jejunostomy was complicated by post-operative vomiting for 2 days, which fixed by conservative treatment.

After SL, 6 cases were operable, one of them displayed small hepatic focal lesion in segment 2 and laparoscopic liver biopsy was performed and frozen section examination revealed bile duct hamartoma. One case was inoperable and showed 3 hepatic focal lesions, porta-hepatisand celiac nodes and mild ascitis (aspiration cytology was taken).

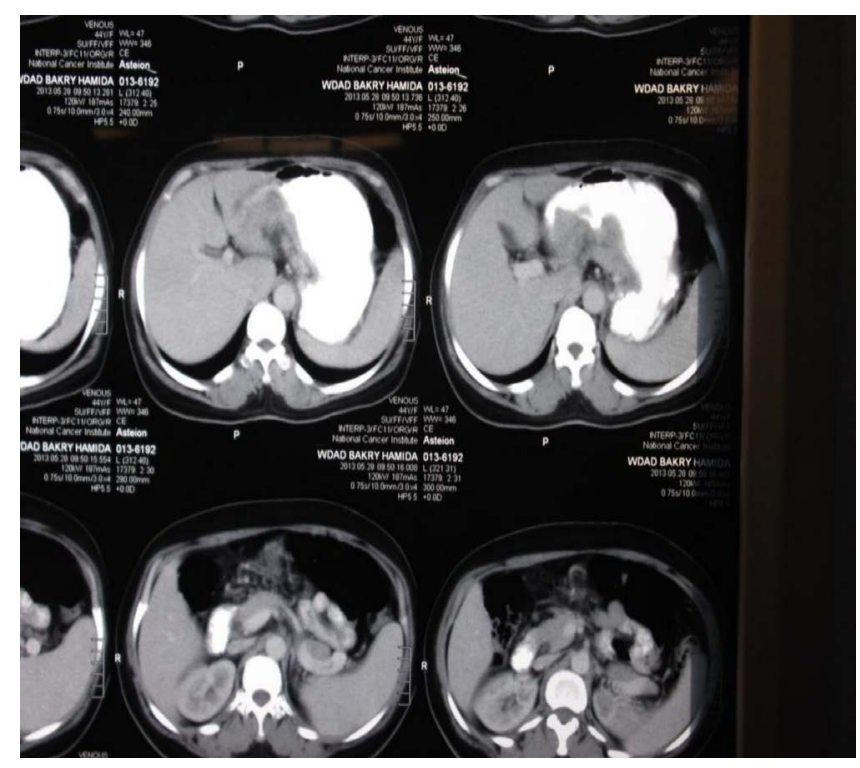

Figure 1. Pre-operative CT scan of gastric carcinoma case without ascites or peritoneal deposit. 


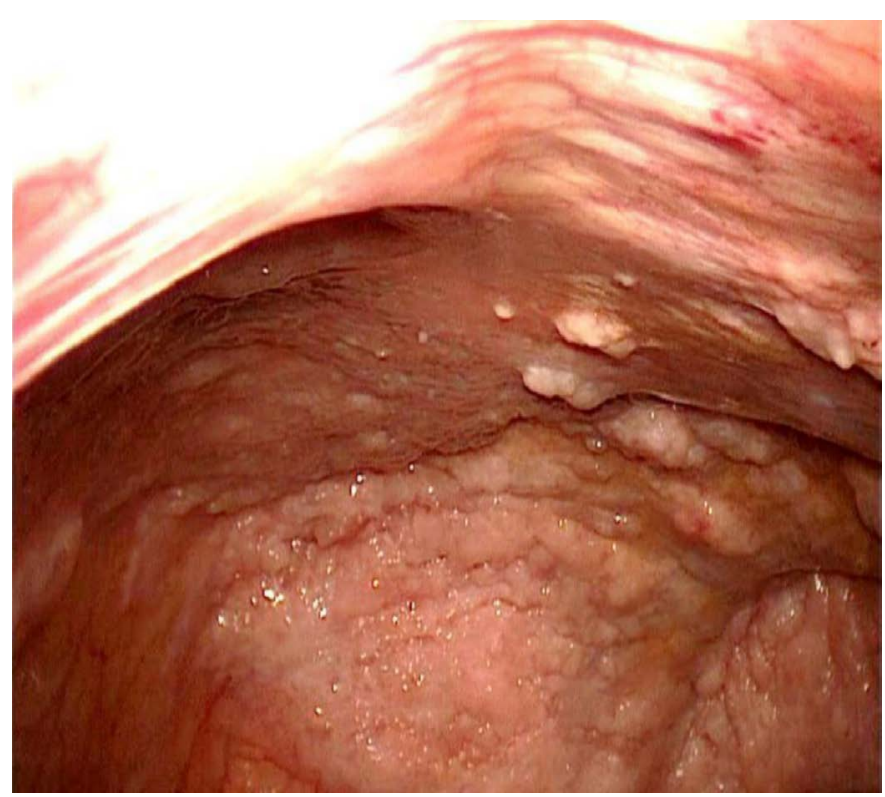

Figure 2. Peritoneal deposit in gastric carcinoma case after DL.

\section{Discussion}

As other invasive analytical measures, SL should be only accomplished when there are no accessible non-invasive staging methods with comparable or equivalent data. [7] Diagnostic profit and the data delivered by SL is essential and capable of altering the management plan, depending on SL results; so therapeutic decisions should be reserved by oncologic multidisciplinary commands and the treatment alternatives, should be obviously specified.

In our work we used LUS shared with standard DL in all our patients to get the full advantage from the technique. The information collected during staging laparoscopy SL were established after examination of operable cases and were accurate except in only 4 out of 62 cases, giving staging laparoscopy and LUS procedure specificity $(77.8 \%)$, positive predictive value (91.8), sensitivity $(100 \%)$ and (100\%) negative predictive value.

Other studies have appraised the additive advantage of laparoscopic US at the period of laparoscopic staging. In a cohort of 90 subjects with pancreatic cancer, laparoscopic US concluded the resectability in 13 patients (14\%), where laparoscopic analysis alone had led to vagueresults [8].

The mutual use of laparoscopy and laparoscopic US confirmed a positive predictive index of $100 \%$, negative predictive index of $98 \%$ and accuracy of $98 \%$. [8] Moreover, Hunerbein et al. [9] stated that laparoscopic US added additional data to laparoscopy in $12 \%$ of subjects with pancreatic cancer, who were establish to have resectable disease on preoperative US, CT and MRI. The mixture of SL with US in this work confirmed irresectability in $57 \%$ of cases with a consequential change in surgical plan. In our series, 14 patients (22.5\%) were inoperable and protected from needless laparotomy after DL, and 48 subjects (77.5\%) were operable.

In a series from the Memorial Sloan-Kettering Cancer Center of 115 patients 
who were all thought resectable based on pre-operative imaging, laparoscopy recognized $41(36 \%)$ patients with irresectable disease who were saved laparotomy. Additionally, the extended laparoscopy technique that the authors of this study used predicted resectability in $92 \%$ of subjects [5].

In another study from the Massachusetts General Hospital that included 125 pancreatic cancer patients who had experienced spiral CT for valuation of resectability, SL recognized $31 \%$ of patients with unsuspected incurable disease and saved them the morbidity of laparotomy. The sensitivity of SL was calculated at $97 \%$ and the specificity at $100 \%$ [10].

Further evidence on the importance of SL comes from a recent study of $84 \mathrm{pa}-$ tients with resectable pancreatic cancer based on preoperative helical CT scan. In this cohort study, staging laparoscopy revealed unpredicted CT-occult metastases in $31 \%$ of patients who were spared laparotomy [11].

The established criteria of inoperability in our series were; ascitis in 9 cases (14.5\%), nodal deposits in 8 cases (12.9\%), liver deposits in 5 cases (8.1\%), peritoneal deposits in one case (1.6\%) and omental nodule in one case (1.6\%). Mutual criteriaoccurred in many of these inoperable cases. Warshaw [12] revealed that small hepatic or peritoneal embeds could be noticed with substantial precision by standard laparoscopy.

In our series, peritoneal wash and cytology was completed in all cases without ascitis; 53 cases (85.5\%) and all of them were negative for malignant cells.

Jimenez et al. [10] described a 7\% rate of positive peritoneal cytology as the only confirmation of metastatic disease during laparoscopy in 125 subjects considered resectable by pre-operative CT scan. The same authors described a $14 \%$ rate of positive peritoneal cytology in the lack of evident metastasis in a larger number of subjects $(\mathrm{n}=239)$ [13].

Moreover, most studies propose that pancreatic cancer cases with positive peritoneal cytology have comparable outcomes to cases with metastatic disease; this is reproduced in the $6^{\text {th }}$ edition of the American Joint Commission on Cancer (AJCC) Cancer Staging Manual, which describes positive peritoneal cytology as M1 disease [14].

So the role of peritoneal wash cytology as auxiliary tool to SL is still debatable taking in attention the low profit of the procedure, elongation of the duration of the technique and cost benefit. In our study, minor complications were met in only 3 cases $(4.8 \%)$, all of them were exposed to palliative management. The other cases with only DL technique showed uneventful post-operative course.

\section{Conclusions}

Regardless of the technological improvements of imaging modalities, yet, recent series stay to document a high incidence of irresectability found at the time of surgery. Low volume metastatic disease and retroperitoneal vascular invasion impeding curative resection are the main causes for the letdown of non-invasive imaging modalities in expecting resectability. The role of laparoscopic staging lies in recognizing those cases with imaging occult irresectable disease, and thus 
in avoiding the morbidity of avoidable laparotomies. Laparoscopic US, and to a lesser extent peritoneal cytology, done at the time of SL, expands its diagnostic precision by letting the identification of irresectable disease (deep hepatic metastases or vascular invasion) overlooked by visual inspection alone.

Staging laparoscopy is clearly helpful for a percentage of cases with upper gastro-intestinal carcinomas and designated in all cases with vague results on preoperative imaging and tumor size more than $\mathrm{T} 1$. We were able to show that DL might help us to avoid needless laparotomy in $22.5 \%$ of patients, avoiding its post-operative complications and reducing post-operative hospital stay and cost.

DL might also help us to accomplish palliative management for the advanced cases, either in the form of palliative resection or bypass or palliative laparoscopic celiac plexus block.

We recommend the use of DL as a safe, effective and complimentary method to the other tedious imaging modalities, in correct staging and probable palliation for upper gastrointestinal malignancies.

\section{References}

[1] Chang, L., Stefanidis, D., Richardson, W.S., Earle, D.B. and Fanelli, R.D. (2009) The Role of Staging Laparoscopy for Intra Abdominal Cancers: An Evidence Based Review. Surgical Endoscopy, 23, 231-241. https://doi.org/10.1007/s00464-008-0099-2

[2] Nieveen van Dijkum, E.J., de Wit, L.T., van Delden, O.M., Rauws, E.A., van Lanschot, J.J., Obertop, H., et al. (1997) The Efficacy of Laparoscopic Staging in Patients with Upper Gastrointestinal Tumors. Cancer, 79, 1315-1319.

https://doi.org/10.1002/(SICI)1097-0142(19970401)79:7<1315::AID-CNCR7>3.0.C O;2-F

[3] Clements, D.M., Bowrey, D.J. and Havard, T.J. (2004) The Role of Staging Investigations for Oesophago-Gastric Carcinoma. European Journal of Surgical Oncology, 30, 309-312. https://doi.org/10.1016/j.ejso.2003.11.013

[4] Luque-de Leon, E., Tsiotos, G.G., Balsiger, B., Barnwell, J., Burgart, L.J. and Sarr, M.G. (1999) Staging Laparoscopy for Pancreatic Cancer Should Be Used to Select the Best Means of Palliation and Not Only to Maximize the Resectability Rate. Journal of Gastrointestinal Surgery, 3, 111-117.

[5] Conlon, K.C., Doughtery, E., Klimstra, D.S., Coit, D.G., Turnbull, A.D. and Brennan, M.F. (1996) The Value of Minimal Access Surgery in the Staging of Patients with Potentially Resectable Peripancreatic Malignancy. Annals of Surgery, 223, 134 140. https://doi.org/10.1097/00000658-199602000-00004

[6] Yan, B.M. and Myers, R.P. (2007) Neurolytic Celiac Plexus Block for Pain Control in Unresectable Pancreatic Cancer. The American Journal of Gastroenterology, 102, 430-438. https://doi.org/10.1111/j.1572-0241.2006.00967.x

[7] Hori, Y. and SAGES Guidelines Committee (2008) Diagnostic Laparoscopy Guidelines. Surgical Endoscopy, 22, 1353-1383.

https://doi.org/10.1007/s00464-008-9759-5

[8] Minnard, E.A., Conlon, K.C., Hoos, A., Dougherty, E.C., Hann, L.E. and Brennan, M.F. (1998) Laparoscopic Ultrasound Enhances Standard Laparoscopy in the Staging of Pancreatic Cancer. Annals of Surgery, 228, 182-187.

https://doi.org/10.1097/00000658-199808000-00006

[9] Hunerbein, M., Rau, B., Hohenberger, P. and Schlag, P.M. (2001) Value of Lapa- 
roscopic Ultrasound for Staging of Gastrointestinal Tumors. Der Chirurg, 72, 914919.

[10] Jimenez, R.E., Warshaw, A.L., Rattner, D.W., Willett, C.G., McGrath, D. and Fernandez-del Castillo, C. (2000) Impact of Laparoscopic Staging in the Treatment of Pancreatic Cancer. Archives of Surgery, 135, 409-414.

https://doi.org/10.1001/archsurg.135.4.409

[11] Vollmer, C.M., Drebin, J.A., Middleton, W.D., Teefey, S.A., Linehan, D.C., Soper, N.J., et al. (2002) Utility of Staging Laparoscopy in Subsets of Peripancreatic and Biliary Malignancies. Annals of Surgery, 235, 1-7.

[12] Warshaw, A.L., Gu, Z.-Y., Wittenberg, J. and Waltman, A.C. (1990) Preoperative Staging and Assessment of Resectability of Pancreatic Cancer. Archives of Surgery, $125,230-233$.

[13] Jimenez, R.E., Warshaw, A.L. and Fernandez-Del Castillo, C. (2000) Laparoscopy and Peritoneal Cytology in the Staging of Pancreatic Cancer. Journal of Hepato-Biliary-Pancreatic Surgery, 7, 15-20. https://doi.org/10.1007/s005340050148

[14] American Joint Commission on Cancer (2002) Exocrine Pancreas. In Greene, F.L., Page, D.L., Fleming, I.D., Fritz, A.G., Balch, C.M., Haller, D.G., et al., Eds., AJCC Cancer Staging Manual, 6th Edition, Springer-Verlag, New York, 157-164. https://doi.org/10.1007/978-1-4757-3656-4_18

\section{Submit or recommend next manuscript to SCIRP and we will provide best service for you:}

Accepting pre-submission inquiries through Email, Facebook, LinkedIn, Twitter, etc. A wide selection of journals (inclusive of 9 subjects, more than 200 journals)

Providing 24-hour high-quality service

User-friendly online submission system

Fair and swift peer-review system

Efficient typesetting and proofreading procedure

Display of the result of downloads and visits, as well as the number of cited articles

Maximum dissemination of your research work

Submit your manuscript at: http://papersubmission.scirp.org/

Or contact jct@scirp.org 